\title{
COMPARISON AND OSCILLATION THEOREMS FOR MATRIX DIFFERENTIAL INEQUALITIES
}

\author{
BY \\ E. S. NOUSSAIR
}

\begin{abstract}
Strong comparison theorems of Sturm's type are established for systems of second order quasilinear elliptic partial differential equations. The technique used leads to new oscillation and nonoscillation criteria for such systems. Some criteria are deduced from a comparison theorem, and others are derived by a direct variational method. Some of our results constitute extensions of known theorems to nonselfadjoint quasilinear systems.
\end{abstract}

1. Introduction. The matrix differential operator $L$ defined by

$$
\begin{aligned}
L V=-\sum_{i, j=1}^{n} D_{i}\left(A_{i j}(x, V) D_{j} V\right)+2 \sum_{i=1}^{n} B_{i}(x, V) D_{j} V+C(x, V) V, \\
A_{i j}=A_{j i}(i, j=1,2, \ldots, n),
\end{aligned}
$$

will be considered for $x \in G, V \in H^{m}$, where $G$ is a nonempty regular bounded domain of $n$-dimensional Euclidean space $E^{n}$, and $H$ is a domain in $E^{m}$ containing the origin. The coefficients $A_{i j}(x, \xi), B_{i}(x, \xi)$ and $C(x, \xi), i, j=1,2, \ldots, n$, are $m \times m$ symmetric matrix functions of class $C^{1}\left(\bar{G} \times H^{m}\right)$, and the $m n \times m n$ matrix $\left(A_{i j}\right)$ is positive definite in $G \times H^{m}$.

A strong theorem of Sturm's type for a quasilinear selfadjoint elliptic system of partial differential equations was obtained recently by C. A. Swanson [8], extending results of Kuks [6], J. B. Diaz and J. R. McLaughlin [2]. The purpose of the present work is to extend Swanson's theorem to nonselfadjoint systems and to obtain oscillation and nonoscillation theorems for such systems. Some of the oscillation theorems were derived by a direct variational method similar to that used in [9].

Points of $E^{n}$ will be denoted by $x=\left(x_{1}, x_{2}, \ldots, x_{n}\right)$ and differentiation with respect to $x_{i}$ by $D_{i}$. The differential operator $l$ defined by

$$
\begin{aligned}
l u=-\sum_{i, j=1}^{n} D_{i}\left[a_{i j}(x, u) D_{j} u\right]+2 \sum_{i=1}^{n} b_{i}(x, u) D_{i} u+c(x, u) u, & \\
& a_{i j}=a_{j i}(i, j=1,2, \ldots, n),
\end{aligned}
$$

will be considered for $x \in G, u \in H$. The coefficients $a_{i j}, b_{i}, c$ are real symmetric

Received by the editors August 31, 1970.

AMS 1969 subject classifications. Primary 3442, 3445, 3490.

Key words and phrases. Matrix differential inequality, oscillatory differential system, oscillation and nonoscillation criteria, strongly oscillatory equation, positive definite matrix. 
functions of class $C^{1}(\bar{G} \times H)$, where $\bar{G}$ is the closure of $G$ in the Euclidean topology of $E^{n}$, and the $m n \times m n$ matrix $\left(a_{i j}(x, u)\right)(i, j=1,2, \ldots, n)$ is positive definite in $G \times H$.

The domain $D$ of $l$ is defined as the set of all functions $u \in C^{2}(G) \cap C^{1}(\bar{G})$ with range in $H$.

The notation $D^{m}$ will be used for the set of all $m \times m$ matrix functions whose column vectors $v_{i} \in D, i=1,2, \ldots, m$. The conclusion of the comparison theorems below concerns matrices $V \in D^{m}$ with the property that $V^{T} L V$ is positive semidefinite.

2. Comparison theorems for $L$. If the $m n \times m n$ matrix

$$
\left(A_{i j}(x, \xi)\right) \quad(i, j=1,2, \ldots, n)
$$

is positive definite for all $(x, \xi) \in G \times H^{m}$, then a diagonal matrix $h(x, \xi)=\left(h_{i i}(x, \xi)\right)$ can be constructed such that for any $(x, \xi) \in G \times H^{m}$, the matrix

$$
M(x, \xi)=\left[\begin{array}{cc}
\left(A_{i j}(x, \xi)\right. & \left(B_{i}(x, \xi)\right)^{T} \\
\left(B_{i}(x, \xi)\right) & h(x, \xi)
\end{array}\right]
$$

is positive definite in $G \times H^{m}$, where $\left(B_{i}\right)^{T}$ denotes the transpose of the $n m \times m$ matrix $\left(B_{i}\right)$. This can be shown easily by using a criterion of Gantmacher [3], and a simple inductive argument.

Let $f[u], F[u, V]$ be the functionals defined by

$$
\begin{aligned}
& f[u]=\int_{G}\left[\sum_{i, j=1}^{n}\left(D_{i} u\right)^{T} a_{i j}(x, u) D_{j} u\right.\left.+2 \sum_{i=1}^{n}\left(D_{i} u\right)^{T} b_{i}(x, u) u+u^{T} c(x, u) u\right] d x \\
& F[u, V]=\int_{G}\left[\sum_{i, j=1}^{n}\left(D_{i} u\right)^{T} A_{i j}(x, V) D_{j} u\right. \\
&\left.+2 \sum_{i=1}^{n}\left(D_{i} u^{T}\right) B_{i}(x, V) u+u^{T}(C(x, V)+h(x, V)) u\right] d x
\end{aligned}
$$

with domains $D_{f}, D_{f} \times D^{m}$, respectively, where $D_{f}$ denotes the set of all vector functions $u \in C^{1}(\bar{G})$ with range in $H$ such that $u(x)$ vanishes identically on $\partial G$, where $\partial G$ is the boundary of $G$.

Following C. A. Swanson [8] we call a matrix $V$ a conjugate matrix relative to $L$ iff $Y_{i}(x, V)=0$ identically in $G$ for $i=1,2, \ldots, n$, where

$$
Y_{i}(x, V)=\sum_{j=1}^{n}\left[V^{T} A_{i j}(x, V) D_{j} V-\left(D_{j} V\right)^{T} A_{i j}(x, V) V\right] .
$$

We shall discuss later the existence of a conjugate matrix.

The first comparison theorem requires that $\partial G$ is only piecewise $C^{1}$. 
THEOREM 1. If

(i) there exists a nontrivial function $u \in D_{f}$ such that $f[u] \leqq 0$;

(ii) $V \in D^{m}$ is a conjugate matrix such that $V^{T} L V$ is positive semidefinite in $G$; and (iii) $f[u] \geqq F[u, V]$;

then det $V(x)$ must vanish at some point in $\bar{G}$.

Proof. Suppose to the contrary that $V(x)$ is nonsingular for all $x \in \bar{G}$. Then there exists a unique $w \in C^{1}(\bar{G})$ satisfying $u(x)=V(x) w(x)$ identically in $\bar{G}$. An easy calculation yields the following identity:

$$
\begin{gathered}
\sum_{i, j=1}^{n}\left(V D_{i} w\right)^{T} A_{i j}(x, V)\left(V D_{j} w\right)+2 \sum_{i=1}^{n}(V w)^{T} B_{i}(x, V) V D_{j} w \\
+(V w)^{T} h(x, V) V w+\sum_{i=1}^{n} D_{i}\left[(V w)^{T} \sum_{j=1}^{n} A_{i j}(x, V)\left(D_{j} V\right) w\right] \\
=F^{+}[u, V]-(V w)^{T}(L V) w+\sum_{i=1}^{n} w^{T} Y_{i}(x, V) D_{i} w
\end{gathered}
$$

where $F^{+}[u, V]$ is the integrand in $F[u, V]$, and $Y_{i}(x, V)$ is defined above.

Since $Y_{i}(x, V)=0$ identically for $i=1,2, \ldots, n, V^{T} L V \geqq 0$ in $G, w(x)=0$ on $\partial G$, and the matrix $M(x, V)$ is positive definite, it follows from integration of the above identity over $G$ and use of Green's identity that $F[u, V] \geqq 0$ with equality iff $V w=0$ identically in $G$.

This is impossible since, by hypothesis, $u=V w$ is a nontrivial function in $D_{f}$. Then the assumption that $V(x)$ is nonsingular throughout $\bar{G}$ leads to the contradiction $f[u] \geqq F[u, V]>0$.

THEOREM 2. Under the hypotheses of Theorem 2, and the further assumption that $\partial G \in C^{1}, \operatorname{det} V(x)$ vanishes at some point in $G$.

Proof. Suppose to the contrary that $V$ is nonsingular throughout $G$. Then there exists a unique $w \in C^{1}$ satisfying $u(x)=V(x) w(x)$ identically in $G$. Since $\partial G$ is of class $C^{1}$, it follows that [1, p. 131] $u \in H_{0}^{1}(G)$, i.e. $u$ belongs to the closure in the norm $\|\cdot\|_{1}$ defined by

$$
\|u\|_{1}^{2}=\int_{G}\left[|u|^{2}+\sum_{i=1}^{n}\left|D_{i} u\right|^{2}\right] d x
$$

of the class $C_{0}^{\infty}(G)$ of infinitely differentiable vector functions with compact support in $G$.

Let $\left\{u_{n}\right\}$ be a sequence of $C_{0}^{\infty}(G)$ functions converging to $u$ in the norm $\|\cdot\|_{1}$. The following inequality follows from identity (3)

$$
\begin{aligned}
F\left[u_{n}, V\right] \geqq \int_{G}\left[\sum_{i, j=1}^{n}\left(V D_{i} w_{n}\right)^{T} A_{i j}(x, V) V D_{j} w_{n}\right. \\
\left.\quad+2 \sum_{i=1}^{n}\left(V D_{i} w_{n}\right)^{T} B_{i}(x, V)\left(V w_{n}\right)+\left(V w_{n}\right)^{T} h(x, V) V w_{n}\right] d x \\
\geqq 0,
\end{aligned}
$$


where $w_{n}$ is the unique solution of $u_{n}(x)=V(x) w_{n}(x)$. Since $A_{i j}(x, V(x)), B_{i}(x, V(x))$, and $C(x, V(x))$ are uniformly bounded in $G$, it follows that

$$
\left|F\left[u_{n}, V\right]-F[u, V]\right| \leqq K\left(\left\|u_{n}\right\|_{1}+\|u\|_{1}\right)\left\|u_{n}-u\right\|_{1},
$$

where $K$ is a positive constant. Since $\left\|u_{n}-u\right\|_{1} \rightarrow 0$ as $n \rightarrow \infty$, we conclude from (4) that $F[u, V] \geqq 0$. If $F[u, V]>0$, we obtain the contradiction $f[u]>0$, and hence $F[u, V]=0$.

Let $S$ denote a ball with $\bar{S} \subset G$, and define

$$
\begin{aligned}
P_{S}\left[u_{n}, V\right]=\int_{S}\left[\left(V D_{i} w_{n}\right)^{T} A_{i j}(\right. & x, V) V D_{j} w_{n} \\
& \left.+2 \sum_{i=1}^{n}\left(V D_{i} w_{n}\right)^{T} B(x, V) V w_{n}+\left(V w_{n}\right)^{T} h(x, V) V w_{n}\right] d x .
\end{aligned}
$$

Then (4) implies

$$
0 \leqq P_{S}\left[u_{n}, V\right] \leqq F\left[u_{n}, V\right],
$$

and by an argument similar to that used in [8] we can show that $w(x)=0$ identically in $S$. Since $S$ is arbitrary, $u(x)=V(x) w(x)=0$ throughout $G$ and hence throughout $\bar{G}$ by continuity. The conclusion of the theorem follows.

When $L$ is symmetric (i.e. $B_{i}=0_{m \times m}$ for all $i=1,2, \ldots, n$ ), we can take $h=0_{m \times m}$, the zero $m \times m$ matrix. The operator $L$ reduces to

$$
L V=-\sum_{i=j=1}^{n} D_{i}\left[A_{i j}(x, V) D_{j} V\right]+C(x, V) V, \quad A_{i j}=A_{j i}
$$

The following theorem is similar to a recent result of Swanson [8]. The latter is obtained if we put $b_{i}=0(i=1,2, \ldots, n)$.

Theorem 3 “Symmetric CASE". If

(i) there exists a nontrivial vector function $u \in D_{f}$ such that $f[u] \leqq 0$;

(ii) $V \in D^{m}$ is a conjugate matrix such that $V^{T} L V$ is positive semidefinite throughout $G$, and

$$
\begin{aligned}
\int_{G}\left[\sum_{i, j=1}^{n} D_{i} u^{T}\left(a_{i j}(x, u)-A_{i j}(x, V)\right) D_{j} u\right. \\
\left.+2 \sum_{i=1}^{n} D_{i} u^{T} b_{i}(x, u) u+u^{T}(c(x, u)-C(x, V)) u\right]^{\sim} d x \geqq 0
\end{aligned}
$$

then either det $V(x)$ vanishes at some point in $G$ or there exists a constant vector $e \neq 0$ such that $u(x)=V(x) e$.

Proof. The proof is similar to that of Theorem 2.

REMARKS. If $m=1$, Theorem 3 extends results of Kreith [5], and Diaz and McLaughlin [2] to quasilinear nonselfadjoint differential inequalities.

If $n=1$, Theorem 4 extends a result of Morse [7]. 
The conclusions in the above theorems apply to conjugate matrices. We show by a simple example that the condition of being a conjugate matrix is necessary:

Let $l u=u^{\prime \prime}+u, L V=V^{\prime \prime}+V$; take

$$
u=\left[\begin{array}{l}
\sin x \\
\sin x
\end{array}\right]
$$

so $u(0)=u(\pi)=0$; let

$$
V=\left[\begin{array}{cc}
\cos x & \sin x \\
-\sin x & \cos x
\end{array}\right]
$$

Then $\operatorname{det} V=1$.

But $V^{T} L V=0, l u=0$, and conditions (i), (ii), (iii) of Theorem 3 are satisfied, except that $V$ is not a conjugate matrix.

3. The existence of a conjugate matrix. We shall show that a conjugate matrix always exists for several nontrivial cases of the operator $L$.

For $n=1$, the definition (1) of $L$ reduces to

$$
L V=-\left(A(x, V) V^{\prime}\right)^{\prime}+2 B(x, V) V^{\prime}+C(x, V) V \quad(x \in I),
$$

where $I$ is some interval.

A matrix $V$ is a conjugate matrix relative to $L$ if $Y(x, V)=0$ identically in $I$, where $Y(x, V)=V^{T} A(x, V) V^{\prime}-V^{\prime T} A(x, V) V$.

Proposition 4. If $V_{0}$ is a solution of the system

$$
\begin{aligned}
L V & =0 & & \text { in } I, \\
Y\left(x_{0}, V\left(x_{0}\right)\right) & =0, & & x_{0} \in I,
\end{aligned}
$$

then any one of the conditions (i), (ii), (iii), (iv) given below implies that $V_{0}$ is a conjugate matrix for $L$;

(i) $B(x, V(x))=0$ identically in I,

(ii) $B A^{-1}$ commutes with $V_{0}$ and $V_{0}^{\prime}$,

(iii) $B$ is a diagonal matrix, $A^{\prime}(x, V(x))$ exists, and $A$ commutes with $V_{0}$ and $V_{0}^{\prime}$, where $A^{\prime} \equiv d A / d x$,

(iv) $A$ is a diagonal matrix, $A^{\prime}\left(x, V_{0}(x)\right)$ exists, and $B$ commutes with $V_{0}$ and $V_{0}^{\prime}$.

Proof. If condition (i) holds, then it follows from the symmetry of the matrices involved that $Y^{\prime}\left(x, V_{0}(x)\right) \equiv 0$ identically in $I$. Since $Y\left(x_{0}, V_{0}\left(x_{0}\right)\right)=0$ by hypothesis, it follows that $Y\left(x, V_{0}(x)\right) \equiv 0$ identically in $I$.

If condition (ii) holds, then

$$
\begin{aligned}
Y^{\prime}\left(x, V_{0}\right) & =2\left[V_{0}^{T} B\left(x, V_{0}\right) V_{0}^{\prime}-V_{0}^{T} B\left(x, V_{0}\right) V_{0}\right] \\
& =2 B\left(x, V_{0}\right) A^{-1}\left(x, V_{0}\right) Y\left(x, V_{0}\right) .
\end{aligned}
$$


Hence $Y\left(x, V_{0}(x)\right)=\left[\exp \int_{0}^{x} B\left(t, V_{0}(t)\right) A^{-1}\left(t, V_{0}(t)\right) d t\right] N$, where $N$ is a constant $m \times m$ matrix. Using the hypothesis on $V_{0}$, we conclude that $U\left(x, V_{0}\right) \equiv 0$ identically in $I$.

If condition (iii) or (iv) holds, we can use a similar argument to show that $Y\left(x, V_{0}\right) \equiv 0$ identically in $I$.

If the operator $L$ defined by (1) is linear, and if we further assume that $C(x)$ $=C\left(x_{i}\right), B_{i}(x)=B\left(x_{i}\right)$, and $A_{i i}(x)=A_{i i}\left(x_{i}\right),\left(\partial / \partial x_{j}\right)\left(A_{j i}\right)=0(i \neq j)$ for at least one suffix $i$, then a conjugate matrix $V(x)$ of $L$ can be sought in the form of a matrix $V(x)=V\left(x_{i}\right)$, where $V\left(x_{i}\right)$ is a conjugate matrix relative to the system of ordinary differential equations

$$
-\frac{d}{d x_{i}}\left(A_{i i}\left(x_{i}\right) \frac{d V}{d x_{i}}\right)+2 B\left(x_{i}\right) \frac{d V}{d x_{i}}+C\left(x_{i}\right) V=0 .
$$

In particular, for strongly elliptic systems with constant coefficients without mixed derivatives, a conjugate matrix exists.

4. Oscillation criteria. We shall find conditions on the coefficients of the operator $L$ defined by (1) which imply that the matrix differential inequality $V^{T} L V \geqq 0$ (as a form) is oscillatory.

The operator $L$ is defined in an unbounded domain $R$ of $E^{n}$. For simplicity we assume that $R$ coincides with $E^{n}$.

NotATION. $R_{r}=\left\{x \in E^{n}:|x|>r\right\}$.

Definition. The matrix differential inequality $V^{T} L V \geqq 0$ (as a form) is said to be oscillatory in $E^{n}$ iff every conjugate solution $V$ of the inequality has the property that det $V(x)$ vanishes at some point in $R_{r}$ for all $r>0$.

Definition. Two functions $\phi, \psi$ of class $C^{1}(0, \infty)$ are called majorants of the matrices $\left(A_{i j}\right)$ and $C$, respectively, if there exists a $C^{1}(0, \infty)$ positive function $\delta$ such that

$$
\begin{aligned}
& \phi(r) \geqq \max _{|x|=r} \sup _{\xi \in H^{m}}[\lambda(x, \xi)]+2 \delta(r), \\
& \psi(r) \geqq \max _{k} \max _{|x|=r} \sup _{\xi \in H^{m}}\left[\sum_{i, j=1}^{m} \frac{1}{\delta}\left(B_{i}^{j k}(x, \xi)\right)^{2}+\mu(x, \xi)\right]+\delta,
\end{aligned}
$$

where $B_{i}(x, \xi)=\left(B_{i}^{j k}(x, \xi)\right)(j, k=1,2, \ldots, m)(i=1,2, \ldots, n), \lambda(x, \xi)$ denotes the largest eigenvalue of the $m n \times m n$ matrix $\left(A_{i j}(x, \xi)\right)(i, j=1,2, \ldots, n)$, and $\mu(x, \xi)$ denotes the largest eigenvalue of the matrix $(C+h)(x, \xi)$ defined before.

Let $W(x), M_{c}(x, \xi)$ denote the $m n \times m n$ matrix functions

$$
\begin{aligned}
W(x) & =\left[\begin{array}{cc}
\phi(r) I_{m n} & \theta^{T} \\
\theta & \psi(r) I_{m}
\end{array}\right] \quad(r=|x|), \\
M_{C}(x, \xi) & =\left[\begin{array}{cc}
\left(A_{i j}(x, \xi)\right) & \left(B_{i}(x, \xi)\right)^{T} \\
B_{i}(x, \xi) & h(x, \xi)+C(x, \xi)
\end{array}\right],
\end{aligned}
$$


respectively, where $I_{m n}, I_{m}$ denote the $m n \times m n$ and the $m \times m$ identity matrices, respectively.

Let $\varphi, \psi$ be any two majorants of $\left(A_{i j}\right), C$, respectively. Let $l_{1}$ be the scalar operator defined by

$$
l_{1} v=-\sum_{i=1}^{n} D_{i}\left[\phi(r) D_{i} v\right]+\psi(r) v \quad(r=|x|) .
$$

Definition. A bounded domain $N$ is a nodal domain for $l_{1}$ iff there exists a nontrivial function $v$ such that $l_{1} v=0$ in $N$ and $v=0$ on $\partial N$.

The operator $l_{1}$ is said to be strongly oscillatory in $E^{n}$ iff it has a nodal domain with $C^{1}$ boundary in the complement of every ball $S$.

THEOREM 5. If $l_{1}$ is strongly oscillatory in $E^{n}$, then the matrix differential inequality $V^{T} L V \geqq 0$ is oscillatory in $E^{n}$.

Proof. Given $r>0$, we choose a nodal domain $N \subset R_{r}$, and a nontrivial function $v$ such that $l_{1} v=0$ in $N$, and $v=0$ on $\partial N$.

Let $l_{0}$ be the differential operator defined by

$$
l_{0} u=-\sum_{i=1}^{n} D_{i}\left[\phi(r) I_{m} D_{i} u\right]+\psi(r) I_{m} u
$$

where $u \in H$, and $I_{m}$ is the $m \times m$ identity matrix, and let

$$
f_{0}[u]=\int_{N}\left[\sum_{i=1}^{n}\left(D_{i} u\right)^{T} \phi(r) I_{m} D_{i} u+u^{T} \psi(r) I_{m} u\right] d x .
$$

If we choose $u$ to be the $m$-vector with each component equal to $v$, then $l_{0} u=0$, and $f_{0}[u]=0$.

Let $V$ be any conjugate matrix such that $V^{T} L V \geqq 0$. We have to show that det $V$ vanishes somewhere in $N$.

A simple calculation shows that the matrix $W(x)-M_{C}(x, \xi)$ is positive definite for every $(x, \xi) \in E^{n} \times H^{m}$. It follows that $F[u, V]<f_{0}[u]$. From the above considerations, it is clear that conditions (i), (ii) and (iii) of Theorem 2 are satisfied. Hence $\operatorname{det} V=0$ in $N$.

Theorem 5 enables us to extend all known oscillation criteria for the scalar operator $l_{1}$ to the matrix differential inequality $V^{T} L V \geqq 0$.

COROllary 6. Let $\phi(r)$ be bounded in $0<r<\infty$ with upper bound $K$. Then the differential inequality $V^{T} L V \geqq 0$ is oscillatory in $E^{n}$ if

$$
\limsup _{r \rightarrow \infty} r^{2} \psi(r)<-K(n-2)^{2} / 4
$$

Proof. The hypotheses imply that $l_{1}$ is strongly oscillatory in $E^{n}$. Corollary 7 is now an immediate consequence of Theorem 6.

Corollary 7 generalizes the Kneser-Hille Theorem [4] to matrix differential inequalities. 
Definitions AND Notations. Let

$$
\begin{aligned}
& A_{i j}(x, V)=\left(A_{i j}^{k l}(x, V)\right), B_{i}(x, V)=\left(B_{i}^{k l}(x, V)\right), \\
& C(x, V)=\left(C^{k l}(x, V)\right), \quad \text { and } \quad h(x, V)=\left(h^{l l}(x, V)\right), \\
& \\
& i, j=1,2, \ldots, n ; k, l=1,2, \ldots, m .
\end{aligned}
$$

Define

$$
\begin{array}{ll}
\tilde{A}_{i j}^{k l}\left(r, \theta_{1}, \ldots, \theta_{n-1}\right)=A_{i j}^{k l}(x, V(x)), & \tilde{B}_{i}^{k l}\left(r, \theta_{1}, \ldots, \theta_{n-1}\right)=B_{i}^{k l}(x, V(x)), \\
\tilde{C}^{k l}\left(r, \theta_{1}, \ldots, \theta_{n-1}\right)=C^{k l}(x, V(x)), & \tilde{h}^{l l}\left(r, \theta_{1}, \ldots, \theta_{n-1}\right)=h^{l l}(x, V(x)),
\end{array}
$$

where $r, \theta_{1}, \ldots, \theta_{n-1}$ are hyperspherical polar coordinates. Let

$$
\begin{aligned}
& \alpha^{l l}(r)=\int_{\omega_{n}} \sum_{i, j=1}^{n} \tilde{A}_{i j}^{l l}\left(r, \theta_{1}, \ldots, \theta_{n-1}\right) d \omega_{n}, \\
& \beta^{l l}(r)=\int_{\omega_{n}} \sum_{i=1}^{n} \mid \tilde{B}_{i}^{l l}\left(r, \theta_{1}, \ldots, \theta_{n-1}\right) d \omega_{n}, \\
& \gamma^{l l}(r)=\int_{\omega_{n}}\left[C^{l l}\left(r, \theta, \ldots, \theta_{n-1}\right)+\tilde{h}^{l l}\left(r, \theta, \ldots, \theta_{n-1}\right)\right] d \omega_{n},
\end{aligned}
$$

where $\omega_{n}$ is the surface of the unit ball in $E^{n}$.

THEOREM 7. The matrix differential inequality $V^{T} L V \geqq 0$ is oscillatory in $E^{n}$ if there exists an integer $l, 1 \leqq l \leqq m$, and a number $q \leqq 2$ such that the following conditions hold for every matrix $V$ with det $V(x) \neq 0$ for all sufficiently large $x$ :

(1) $\left(1 / b^{q}\right) \int_{b}^{2 b} r^{n-1} \alpha^{l l}(r) d r$ is bounded above for all $b>0$.

(2) $\left(1 / b^{q}\right) \int_{b}^{2 b} r^{n-1}(2 b-r) \beta^{l l}(r) d r$ is bounded above for all $b>0$.

(3) For $a>0, \int_{a}^{\infty} \gamma^{l l}(r) r^{n-1} d r=-\infty$.

Proof. Suppose to the contrary that the differential inequality $V^{T} L V \geqq 0$ is not oscillatory. Then there exists a positive number $a$, and a conjugate matrix $V(x)$ such that $V^{T} L V \geqq 0$, and det $V(x) \neq 0$ for $|x|>a$. Hence a unique solution $w(x)$ of $u(x)=V(x) w(x)$ exists in $R_{a}=\{x:|x|>a\}$ for any $m$-vector function $u(x)$. By integrating identity (3) by parts, and applying Green's formula, we obtain the inequality

$$
\begin{aligned}
& F[u, V]=\int_{a \leqq|x| \leqq 2 b}\left[\sum_{i, j=1}^{n}\left(D_{i} u\right)^{T} A_{i j}(x, V) D_{j} u\right. \\
&\left.+2 \sum_{i=1}^{n}\left(D_{i} u\right)^{T} B_{i}(x, V) u+u^{T}(C(x, V)+h(x, v)) u\right] d x
\end{aligned}
$$

$\geqq 0$,

for any piecewise $C^{1}$ function $u(x)$ on the annulus $N=\{x: a \leqq|x| \leqq 2 b\}$ such that $u(x)=0$ on the boundary $\partial N$. In particular choose $u$ to be the vector function

$$
\begin{aligned}
& u(r)=0, \quad r \leqq a, \\
& u(r)=(r-a) e_{l}, \quad a<r \leqq a+1, \\
& u(r)=e_{l}, \quad a+1<r \leqq b, \\
& u(r)=(2 b-r) e_{l} / b, \quad b<r \leqq 2 b, \\
& u(r)=0, \quad r>2 b \text {, }
\end{aligned}
$$


where $e_{l}$ is the unit $m$-vector with 1 in the $l$ th position and zero elsewhere. Choose constants $K_{1}, K_{2}$ such that

$$
\begin{aligned}
\frac{1}{b^{q}} \int_{b}^{2 b} r^{n-1} \alpha^{l l}(r) d r \leqq K_{1} & \text { for all } b>0, \\
\frac{1}{b^{q}} \int_{b}^{2 b} r^{n-1}(2 b-r) \beta^{l l}(r) d r \leqq K_{2} & \text { for all } b>0 .
\end{aligned}
$$

This is possible by hypothesis. Then

$$
\begin{aligned}
F[u, V] \leqq & \int_{a}^{a+1} \alpha^{l l}(r) r^{n-1} d r+K_{1} b^{q-2} \\
& +\int_{a}^{a+1}(r-a) \beta^{l l}(r) r^{n-1} d r+K_{2} b^{q-2} \\
& +\int_{a}^{a+1}(r-a)^{2} \gamma^{l l}(r) r^{n-1} d r \\
& +\int_{a+1}^{b} \gamma^{l l}(r) r^{n-1} d r+\int_{b}^{2 b}\left(\frac{2 b-r}{b}\right)^{2} \gamma^{l l}(r) r^{n-1} d r
\end{aligned}
$$

By hypothesis (3) there exists $b_{0}>a+1$ such that the sum of the first six terms of the right member of the above inequality is negative for all $b>b_{0}$.

Define $f(r)=\int_{b_{0}}^{r} \gamma^{l l}(r) r^{n-1} d r$. Then $\lim _{r \rightarrow \infty} f(r)=-\infty$ by hypothesis (3). Now we choose $b \geqq b_{0}$ in the definition of the vector function $u$ as the last root of $f(r)$.

Then

$$
\int_{b}^{2 b}\left(\frac{2 b-r}{b}\right)^{2} \gamma^{l l}(r) r^{n-1} d r=\left[\left(\frac{2 b-r}{b}\right)^{2} f(r)\right]_{b}^{2 b}+\int_{b}^{2 b} \frac{2(2 b-r)}{b^{2}} f(r) d r<0 .
$$

It follows from (8) that $F[u, V]<0$, contradicting (7).

When the operator $L$ is symmetric, i.e. $B_{i} \equiv 0$ for $i=1,2, \ldots, n$, we can choose $h=0$. In this case the following more general version of Theorem 7 holds.

TheOREM 8 "SyMmetric CASE". Under the hypotheses of Theorem 7 with the weaker assumption that the matrix $\left(A_{i j}\right)(i, j=1,2, \ldots, n)$ is positive semidefinite, the matrix differential inequality $V^{T} L V \geqq 0$ is oscillatory in $E^{n}$.

In the case $n=1$, the definition (1) of $L$ reduces to

$$
L V=-\frac{d}{d x}\left(A(x, V) \frac{d}{d x} V\right)+2 B(x, V) \frac{d}{d x} V+C(x, V) V
$$

where $x \in[a, \infty)$. We can use the above method to generalize the Hille-Kneser classical theorem [4]. For simplicity we assume that $A(x, V)=I, B=0$ where $I$ is the identity matrix.

THEOREM 8. The matrix differential inequality $V^{T} L V \geqq 0$ is oscillatory in $E^{n}$ if there exists an integer $l, 1 \leqq l \leqq m$, such that the following conditions hold for every matrix $V$ with det $V(x) \neq 0$ for all sufficiently large $x$ : 
(1) $C^{l l}(x, V(x)) \leqq-1 / 4 x^{2}$;

(2) $\lim \sup _{a \rightarrow \infty} \log a \int_{a}^{\infty} x\left|C^{l l}(x, V(x))+1 / 4 x^{2}\right| d x>1$.

Proof. If the inequality $V^{T} L V \geqq 0$ is not oscillatory, then, as in the proof of Theorem 7, we find a number $b$, and a conjugate matrix $V(x)$ such that $V^{T} L V \geqq 0$, $\operatorname{det} V(x) \neq 0$ for $|x|>a$, and

$$
F[u, V]=\int_{b}^{\infty}\left[u^{T} u+u^{T} C(x, V(x)) u\right] d x \geqq 0
$$

for any $m$-vector function $u$ with compact support in $[b, \infty)$.

Now let $u=x^{1 / 2} v$ and $x=\log t$. Then

$$
F[u, V]=\int_{e^{b}}^{\infty}\left[v^{\prime T} v^{\prime}+v^{T}(1 / 4+\widetilde{C}(t)) v\right]
$$

where $\tilde{C}(t)=x^{2} C(x, V(x))$.

By hypothesis (2), we can choose a sequence $a_{k} \rightarrow \infty$, for which we have for some $\delta>0$ that

$$
\lim _{a_{k} \rightarrow \infty} a_{k} \int_{a_{k}}^{\infty}\left|\tilde{C}^{u l}(t)+1 / 4\right| d t>1+\delta
$$

where $\tilde{C}(t)=\left(\tilde{C}^{i j}(t)\right)(i, j=1,2, \ldots, m)$.

We now choose the vector function $v(t)$ as follows:

$$
\begin{aligned}
& v(t)=0, \quad t \leqq a / 2, \\
& v(t)=((2 t-a) / a) e_{l}, \quad a / 2<t \leqq a, \\
& v(t)=e_{l}, \quad a<t<q, \\
& v(t)=((2 q-t) / q) e_{l}, \quad q<t \leqq 2 q,
\end{aligned}
$$

where $e_{l}$ is the unit $m$-vector with 1 in the $l$ th position, and $a=a_{k}$ is chosen such that $a \int_{a}^{\infty}\left|\tilde{C}^{l l}(t)+1 / 4\right| d t>1+\delta / 2$, and $a / 2>e^{b}$. We then choose $q$ so that $a / q<\delta / 4$. Then

$$
F[u, V] \leqq \frac{1}{a}+\frac{1}{q}-\int_{a}^{\infty}\left|\tilde{C}^{u}(t)+\frac{1}{4}\right| d t<\frac{1}{a}[1+\delta / 4-(1+\delta / 2)]<0,
$$

which contradicts $\left(^{*}\right)$. This proves the theorem.

REMARK. For $n=1$, Theorem 8 is sharper than Kneser's theorem for linear second order equations [4].

5. Nonoscillation criteria. In this section, we obtain a Kneser-Hille [4] nonoscillation criteria for the vector equation

$$
l u=-\sum_{i, j=1}^{n} D_{i}\left[a_{i j}(x, u) D_{j} u\right]+2 \sum_{i=1}^{n} b_{i}(x, u) D_{i} u+c(x, u) u=0
$$


defined in an unbounded domain $R$ of $E^{n}$. No restrictions are required on the shape of the domain $R$. The conditions on the coefficients $a_{i j}, b_{i}, c$ are as given before, and $u \in H$.

Definition. The operator $l$ is said to be nonoscillatory in the domain $R$ if there exists $r>0$ such that the system (9) has no nontrivial solution vanishing on the boundary of any bounded $n$-dimensional domain belonging to $R \cap\{x:|x|>r\}$.

DEFINITION. Let

$$
m(x, \xi)=\left[\begin{array}{cc}
a_{i j}(x, \xi) & \left(b_{i}(x, \xi)\right)^{T} \\
b_{i}(x, \xi) & c(x, \xi)
\end{array}\right]
$$

Let $\lambda$ be the smallest eigenvalue of the $m n \times m n$ matrix $\left(a_{i j}\right), i, j=1,2, \ldots, n$, and $\mu$ be the smallest eigenvalue of the $m \times m$ matrix $c$.

Definition. A function $g$ of class $C^{1}(0, \infty)$ is said to be a minorant of the matrix $c$ if there exists a $C^{1}(0, \infty)$ positive function $t$ such that

$$
g(r) \leqq \min _{1 \leqq k \leqq m} \min _{|x|=r} \inf _{\xi \in H} \sum_{j=1}^{m} \sum_{i=1}^{n}-\frac{1}{2}\left(b_{i}^{j k}(x, \xi)\right)^{2}+t(x, \xi),
$$

where $b_{i}(x, \xi)=\left(b_{i}^{j k}(x, \xi)\right), j, k=1,2, \ldots, m, i=1,2, \ldots, n$.

A function $f \in C^{1}(0, \infty)$ is a minorant of $\left(a_{i j}\right)$ if there exists a $C^{1}(0, \infty)$ positive function $z$ such that

$$
0<f(r) \leqq \min _{|x|=r} \inf _{\xi \in H}[\lambda(x, \xi)-2 z(r)] .
$$

Let

$$
\mathscr{N}(x)=\left[\begin{array}{cc}
f(r) I_{m n} & \theta^{T} \\
\theta & g(r) I_{m}
\end{array}\right] \quad(r=|x|),
$$

where $I_{m n}$ is the $m n \times m n$ identity matrix, $I_{m}$ is the $m \times m$ identity matrix, and $\theta^{T}$ is the $m n \times m$ zero matrix.

THEOREM 9. Suppose the matrices $\left(a_{i j}\right), c$ admit minorants $f, g$ respectively, such that $f$ is bounded below in $R$ by some positive number $\lambda_{0}$. Then the equation (9) is nonoscillatory in $R$ if

$$
\lim _{r \rightarrow \infty} \inf r^{2} g(r)>-(n-2)^{2} \lambda_{0} / 4
$$

Proof. Suppose to the contrary that there exists a bounded $C^{1}$ nodal domain $N_{s}$ and a solution $u_{s}$ of (9) such that $u_{s}=0$ on $\partial N_{s}$. From the hypothesis that $f, g$ are minorants of $\left(a_{i j}\right), c$ respectively, it is easy to show that the matrix $M(x, \xi)-N(x)$ is positive semidefinite for all $(x, \xi) \in E^{n} \times H$. Then an application of Green's formula gives

$$
0=\int_{N_{s}} u_{s} l u_{s} d x \geqq \int_{N_{s}}\left[\sum_{i=1}^{n}\left(D_{i} u_{s}\right)^{T} \lambda_{0} I_{m} D_{i} u_{s}+g(|x|) u^{T} u_{s}\right] d x
$$


Let $u_{s i}, i=1,2, \ldots, m$, denote the $i$ th component of the vector function $u_{s}$. Hence the above inequality implies that

$$
\int_{N_{s}} \sum_{j=1}^{m}\left(\sum_{i=1}^{n} \lambda_{0}\left(D_{i} u_{s j}\right)^{2}+g(r(x)) u_{s j}^{2}\right) d x \leqq 0 .
$$

Let $u_{s j}(x)=\tilde{u}_{s j}\left(r, \theta_{1}, \ldots, \theta_{n-1}\right)$, where $r, \theta_{1}, \ldots, \theta_{n-1}$ are hyperspherical polar coordinates. Extend $\tilde{u}_{s j}$ to all of $R$ such that it is identically zero outside $N_{s}$. We shall denote the extended function by $\tilde{u}_{s j}$. From the representation

$$
\int_{s}^{\infty} r^{n-3}\left(\tilde{u}_{s j}(r, \cdot)\right)^{2} d r=-2 \int_{s}^{\infty} \tilde{u}_{s j}(t, \cdot) \frac{\partial}{\partial t} \tilde{u}_{s j}(t, \cdot) d t \int_{s}^{t} r^{n-3} d r,
$$

and by means of Cauchy-Schwarz inequality we obtain the inequality

$$
\int_{s}^{\infty} r^{n-3}\left(\tilde{u}_{s j}(r, \cdot)\right)^{2} d r \leqq \frac{4}{(n-2)^{2}} \int_{s}^{\infty} r^{n-1}\left(\frac{\partial}{\partial r} \tilde{u}_{s j}\right)^{2} d r
$$

for $j=1,2, \ldots, n$. Integrating the two sides of the above inequality with respect to the angular coordinates $\theta_{1}, \theta_{2}, \ldots, \theta_{n-1}$ we obtain

$$
\int_{N_{s}}\left[\sum_{i=1}^{n}\left(D_{i} u_{s j}\right)^{2}-\frac{(n-2)^{2}}{4 r^{2}} u_{s j}\right] d x \geqq 0
$$

for all $j=1,2, \ldots, m$.

The hypothesis implies that there exists a constant $s_{0}>0$ such that $r^{2} g(r)$ $>-(n-2)^{2} \lambda_{0} / 4$ provided that $r \geqq s_{0}$. Hence, from (11) we have

$$
\int_{N_{s_{0}}}\left[\sum_{i=1}^{n} \lambda_{0}\left(D_{i} u_{s_{0} j}\right)^{2}+g(r(x)) u_{s_{0} j}^{2}\right] d x>0
$$

for all $j=1,2, \ldots, m$. This contradicts (10), and Theorem 9 follows.

REMARK. When the operator $l$ defined by (9) is symmetric, i.e. $b_{i}=0$ for $i=1,2, \ldots, n$, we can choose minorants $f, g$ such that

$$
0<f(r) \leqq \min _{|x|=r} \inf _{\xi \in H} \lambda(x, \xi), \quad g(r) \leqq \inf _{|x|=r} \inf _{\xi \in H} \mu(x, \xi) .
$$

In this case Theorem 9 generalizes the Kneser-Hille nonoscillation criterion [4] to nonlinear partial differential systems.

\section{REFERENCES}

1. S. Agmon, Lectures on elliptic boundary value problems, Van Nostrand Math. Studies, no. 2, Van Nostrand, Princeton, N. J., 1965. MR 31 \#2504.

2. J. B. Diaz and J. R. McLaughlin, Sturm separation and comparison theorems for ordinary and partial differential equations, Atti Accad. Naz. Lincei Mem. Cl. Sci. Fis. Mat. Natur. Sez. I, Matematica, Meccanica, Astronomia, Geodesia e Geofisica, Rome.

3. F. R. Gantmaher, The theory of matrices. Vol. 1, GITTL, Moscow, 1953; English transl., Chelsea, New York, 1959. MR 16, 438; MR 21 \#6372c.

4. E. Hille, Non-oscillation theorems, Trans. Amer. Math. Soc. 64 (1948), 234-252. MR 10, 376. 
5. K. Kreith, A strong comparison theorem for selfadjoint elliptic equations, Proc. Amer. Math. Soc. 19 (1968), 989-990. MR 37 \#3178.

6. L. M. Kuks, Sturm's theorem and the oscillation of solutions of strongly elliptic systems, Dokl. Akad. Nauk SSSR 142 (1962), 32-35=Soviet Math. Dokl. 3 (1962), 24-27. MR 26 \#441.

7. M. Morse, A generalization of the Sturm separation and comparison theorems in n-space, Math. Ann. 103 (1930), 52-69.

8. C. A. Swanson, Comparison theorems for elliptic differential systems, Pacific J. Math. 33 (1970), 445-450.

9. - Oscillation criteria for nonlinear matrix differential inequalities, Proc. Amer. Math. Soc. 24 (1970), 824-827.

UNIVERSity OF British COLUMBIA,

Vancouver, British Columbia, Canada 